\title{
Puesta en marcha de un catálogo de demostraciones experimentales en asignaturas básicas.
}

\author{
Rosario Isabel Vilaplana Cerdáa, Romina Del Rey Tormos ${ }^{a}$, Oscar Gomis Hilario ${ }^{a}$ Jesús \\ Alba Fernández ${ }^{b}$, Francisco Javier Manjón Herrera ${ }^{c}$, Vanessa Paula Cuenca Gotor ${ }^{\mathfrak{c}}$ y \\ Juan Antonio Monsoriu Serrac
}

aUniversitat Politècnica de Valéncia, Escuela Politécnica Superior de Alcoy, Departamento de Física Aplicada, Pz. Ferràndiz i Carbonell s/n - 03801 Alcoy (Alicante).rovilap@fis.upv.es, osgohi@fis.upv.es, roderey@,fis.upv.es

bUniversitat Politècnica de Valéncia, Escuela Politécnica Superior de Gandia, Departamento de Física Aplicada, C/Paraninfo n ${ }^{\circ} 1$ - 46715 Grao de Gandia. jesalba@fis.upv.es

'Universitat Politècnica de Valéncia, Escuela Técnica Superior de Ingeniería del Diseño, Departamento de Física Aplicada, Camino de Vera s/n - 46022 Valencia. fjmanjon@fis.upv.es, vacuego@fis.upv.es, imonsori@fis.upv.es,

\section{Resumen}

En este trabajo se muestran los primeros pasos que se han llevado a cabo dentro de un proyecto PIME de la convocatoria 2016-2017 del Vicerrectorado de Estudios, Calidad y Acreditación de la Universidad Politécnica de Valencia titulado "Metodologías activas en asignaturas básicas. Creación de un catálogo de demostraciones experimentales o proyectos como recursos didácticos para la motivación de título". Este proyecto persigue como objetivo la creación de un catálogo de demostraciones experimentales sencillas, realizadas con materiales fácilmente disponibles y de bajo coste. Se pretende utilizar este recurso en las clases de teoría o problemas para poner de manifiesto fenómenos físicos y su relación con los modelos teóricos que los explican, favoreciendo su comprensión, así como involucrar a los estudiantes en alguna de estas demostraciones experimentales a través de pequeños proyectos. Aqui se presentan los primeros diseños de estas demostraciones experimentales que serán implantadas de forma más generalizada a partir de los siguientes cursos.

Palabras claves: Metodologías Activas, Motivación, Asignaturas Básicas, Competencias, Demostraciones Experimentales, Aprendizaje Basado en Proyectos.

\section{Summary}

We present in this work the first steps carried out within a 2016-2017 PIME of the Vice Chancery for Studies, Quality and Accreditation the Polytechnic University of Valencia entitled "Active methodologies in basic subjects. A 
catalogue of experimental Physics demonstrations designed as teaching resources for the degree motivation". This project aims the design of a catalogue of simple experimental Physics demonstrations made with readily available low cost materials. It is intended to use this resource during lessons of theory and problems to demonstrate physical phenomena and their relationship to the theoretical models, favouring their understanding as well as to involve the students in the design of some of these experimental demonstrations through the implementation of small projects. The first designs of these experimental Physics demonstrations that will be implemented in a more general way the following courses are here presented.

Key words: Active Methodologies, Motivation, Basic Subjects, Competences, Experimental Demonstrations, Project-Based Learning.

\section{Introducción}

En este trabajo se pretende exponer la puesta en marcha del Proyecto de Innovación y Mejora Educativa (PIME) titulado: "Metodologías activas en asignaturas básicas. Creación de un catálogo de demostraciones experimentales o proyectos como recursos didácticos para la motivación de título", que está enfocado a la creación e implementación de un catálogo de demostraciones experimentales de Física.

Las demostraciones experimentales de Física están asociadas al desarrollo de la ciencia moderna y fueron utilizadas desde el siglo XVII en sociedades científicas, academias, salones y conferencias para todo tipo de público. También han sido empleadas de forma generalizada como herramientas docentes en instituciones educativas y universidades constituyéndose como prácticas docentes habituales en diferentes países. A pesar de que su uso está recogido explícitamente en el proyecto europeo "Tuning" (González, Wagenaar, 2003) de Física para la armonización de titulaciones universitarias, en nuestro país no forma parte de las prácticas docentes generalizadas. A este respecto, nos consta que en la Facultad de Ciencias Físicas de la Universitat de València existe un proyecto docente que ha incorporado el uso de las demostraciones experimentales como herramienta habitual en sus clases, y que dicho proyecto ha obtenido resultados altamente satisfactorios (Ferrer 2012). En concreto, a fecha de hoy, ya disponen de una colección que contiene más de 100 demostraciones experimentales de diferentes temas de Física utilizada por más de 30 profesores con docencia en 20 asignaturas de Física de 17 grados diferentes de ciencias, ciencias de la salud e ingeniería, y algunos estudios de máster llegando a involucrar a más de 2000 estudiantes.

Algunos de los estudiantes que llegan a la universidad no han cursado Física durante la enseñanza secundaria y la mayoría de quienes sí lo han hecho no han realizado prácticas de laboratorio o visto demostraciones experimentales, por lo que en general desconocen los fenómenos físicos más básicos. Las demostraciones experimentales o prácticas caseras (Díaz, 2007) se caracterizarán por el uso de materiales fácilmente asequibles y de bajo coste, que los propios alumnos pueden llegar a diseñar. Las demostraciones experimentales pueden ser una herramienta muy útil para transmitir al alumno que detrás de los fenómenos cotidianos

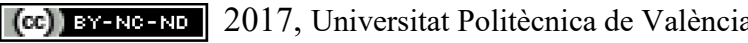


que acontecen se encuentran las leyes físicas y que con el diseño de experimentos sencillos podemos llegar a demostrarlos, entenderlos y estudiarlos con los modelos teóricos que los explican. En otras palabras, con el uso de las demostraciones experimentales se pretende fomentar la capacidad de sorpresa del alumnado ante los fenómenos naturales y mostrarle que detrás de la mayoría de los dispositivos que usamos casi siempre hay una o varias leyes físicas involucradas, llegando a constituir la base de su funcionamiento. De un modo más general, el uso de demostraciones experimentales permite articular un proceso de observación racional en el que se emitan conclusiones en términos de los modelos físicos estudiados en el ámbito teórico, algo esencial en la comprensión del método científico; además de ofrecer la posibilidad de involucrar de forma activa a los alumnos en su propio proceso de aprendizaje.

Es conocido que la calidad de la docencia universitaria en enseñanzas técnicas, así como la mejora del aprendizaje se puede conseguir, entre otros muchos recursos, con metodologías activas (Aparicio, González, Sobrevila, 2005) (Calvo, Lopez-Guede, Zulueta, 2010) (Alba, Torregrosa, del Rey, 2015). Como hemos descrito previamente, la propia naturaleza intrínseca de las demostraciones experimentales hace que sea posible involucrar a los alumnos de forma activa y en primera persona en el proceso de aprendizaje. En este sentido, consideramos que se puede involucrar a los alumnos en el desarrollo de alguna de las demostraciones experimentales con los recursos que se generarán en este PIME haciendo uso de este modo de metodologías activas en el aula. Esto permite pasar de un planteamiento centrado en el profesor a uno más orientado al estudiante. Dicho de otro modo, el propio estudiante, al explicar qué ha diseñado y cómo ha diseñado su experimento, puede pasar a hacer la función del profesor lo que permite desarrollar la empatía de los alumnos hacia el profesorado y tener la oportunidad de trabajar algunas competencias transversales asignadas a las asignaturas de Física.

En este trabajo se muestran los primeros recursos generados en este proyecto, como resultado de la puesta en marcha de la creación del catálogo de demostraciones experimentales. Estos recursos, a fecha de la redacción de este trabajo, todavía no han podido ser aplicados de modo generalizado en el aula, a excepción de algunas demostraciones experimentales y un pequeño proyecto desarrollados en clase (el mostrado en este trabajo a modo de ejemplo). En cualquier caso, estos pocos recursos nos han servido como experiencias piloto y nos han permitido comprobar que los alumnos responden con alto grado de motivación. A partir del siguiente curso, podremos aplicar el catálogo de demostraciones experimentales en el aula de una manera más global y no solamente los recursos presentados en este trabajo. Será entonces cuando verdaderamente podremos evaluar el objetivo perseguido y la metodología aplicada. Así mismo, es de destacar que este método facilitará el desarrollo y la evaluación de algunas de las competencias que la titulación tiene asignadas como punto de control a las asignaturas de Física, que pueden variar dependiendo de la titulación.

\section{Objetivos}

El objetivo de este trabajo es presentar los primeros recursos generados en el proyecto PIME titulado: "Metodologías activas en asignaturas básicas. Creación de un catálogo de 
demostraciones experimentales o proyectos como recursos didácticos para la motivación de título", así como poner de manifiesto el carácter multidisciplinar que puede presentar este tipo de recursos.

El desarrollo de estas demostraciones experimentales, conlleva la generación de nuevos recursos tales como fichas descriptivas, vídeos y cuestionarios que permiten a profesores y estudiantes ver y pensar los fenómenos físicos de forma permanente, más allá del uso instantáneo del material en el aula. Esta colección (o banco) se está desarrollando de forma colaborativa por los profesores que participan en el proyecto PIME. Es conocido que, con las metodologías activas basadas en el PBL (aprendizaje basado en proyectos) la carga del profesor se multiplica (Alba, Torregrosa, del Rey, 2015). Con la creación del catálogo o banco de experimentos, distribuyendo muy bien los esfuerzos, será posible multiplicar los recursos didácticos sin que aumente significativamente la carga del profesor.

El carácter multidisciplinar de este trabajo presenta la ventaja de estar formado por un número importante de profesores de una asignatura básica (Física), de gran importancia en todas las enseñanzas técnicas, que se imparte en distintos grados (Grado en Ingeniería en Diseño Industrial y Diseño del Producto, Grado en Ingeniería Mecánica, Doble Grado en Ingeniería Informática y Administración y Dirección de Empresas y Grado en Ingeniería de Sistemas de Telecomunicación, Sonido e Imagen) de distintas escuelas (Escola Politécnica Superior de Alcoi, Escola Politécnica Superior de Gandia y Escola Técnica Superior d'Enginyeria del Diseny) de los tres campus universitarios de la Universitat Politècnica de València (Campus de Alcoi, Campus de Gandia y Campus de Vera).

Otro objetivo que se pretende más a largo plazo consiste en conseguir involucrar a más profesores en las próximas convocatorias y consolidar un grupo de trabajo de innovación docente con aquellos participantes que demuestren un interés para participar activamente en la consecución de este proyecto inicial.

\subsection{Objetivos específicos}

En este apartado de "objetivos específicos" creemos interesante numerar los objetivos específicos del Proyecto de Innovación y Mejora Educativa al que este trabajo hace referencia:

a) Crear una colección de demostraciones experimentales disponible para el profesorado que imparte docencia en asignaturas de Física para su uso en directo en las clases de teoría y problemas. En el siguiente apartado se muestran imágenes de algunos de los primeros experimentos diseñados para alcanzar el objetivo del proyecto.

b) Desarrollar recursos asociados a las demostraciones como fichas descriptivas, vídeos y cuestionarios disponibles para profesores y estudiantes con el fin de ver y pensar los fenómenos físicos, más allá de los instantes de uso en el aula. En el siguiente apartado, se muestra alguna de las primeras fichas descriptivas a modo de ejemplo.

c) Usar metodologías activas: involucrar a los alumnos en el desarrollo de alguna de estas demostraciones experimentales de modo que ellos tomen más iniciativa y

(cc) EY-NC-ND 2017, Universitat Politècnica de València 
protagonismo, desarrollando por grupos, sus propias demostraciones experimentales tutorizadas por el profesor.

d) Crear rúbricas o describir formas de evaluación adecuadas para evaluar actividades desarrolladas usando demostraciones experimentales o las actividades grupales, de modo que se pueda añadir algún apartado que ayude al profesor en la evaluación de las competencias transversales asignadas como punto de control a las asignaturas de Física en los Grados y/o en los Másteres dentro del Proyecto Institucional de la Comisión de Título de la UPV.

\section{Desarrollo de la innovación}

Dado que la puesta en marcha de la creación del catálogo de demostraciones experimentales y la innovación en sí dentro del aula, todavía no han podido ser aplicadas totalmente, en este apartado mostramos como estamos desarrollando, o creando, los recursos para poder disponer del catálogo de experimentos más adecuado, y optimizando siempre que sea posible, con el esfuerzo del profesorado implicado.

Como somos profesores de tres campus diferentes, estamos usando la herramienta Google Drive como almacén compartido del material ofimático para poder llevar a cabo el desarrollo de este proyecto innovador. En la captura de pantalla (ver figura 1) mostramos la estructura de directorios creada con el fin de que cada profesor pueda ir subiendo las fichas y el material correspondiente a las prácticas demostrativas o actividades grupales que desarrolle y a su vez pueda modificar o corregir ficheros del resto de los profesores sin necesidad de reunirse físicamente.

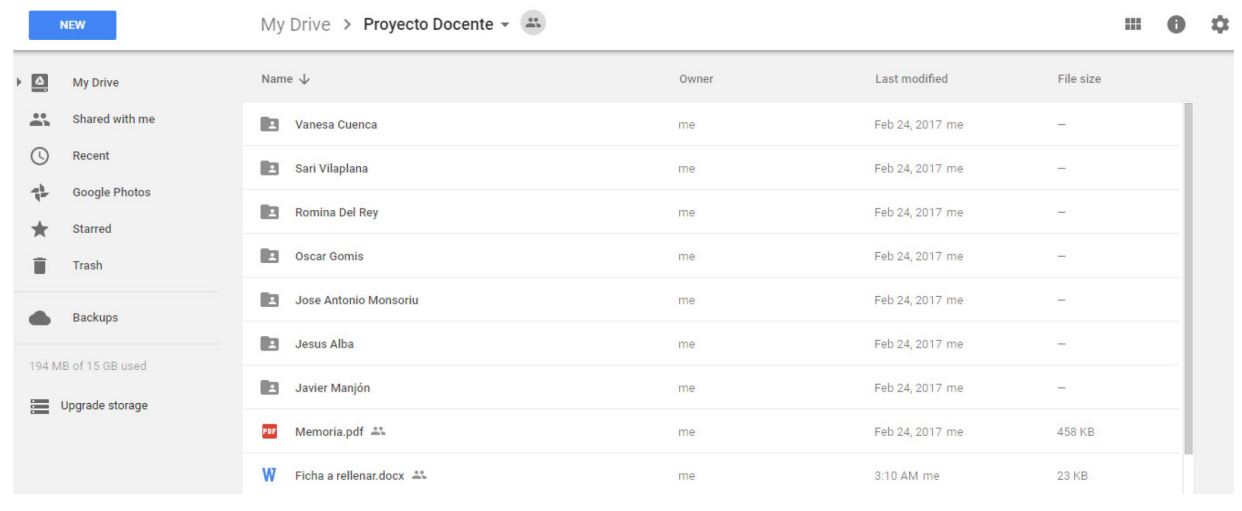

Fig. 1 Captura de pantalla de la carpeta proyecto docente en el Google Drive

Uno de los ficheros clave que se ha definido para que todos sigamos un modelo estándar es el que mostramos en la figura 1 con la imagen de un documento Word de título "Ficha a rellenar". El contenido de este fichero se muestra en la figura 2. 


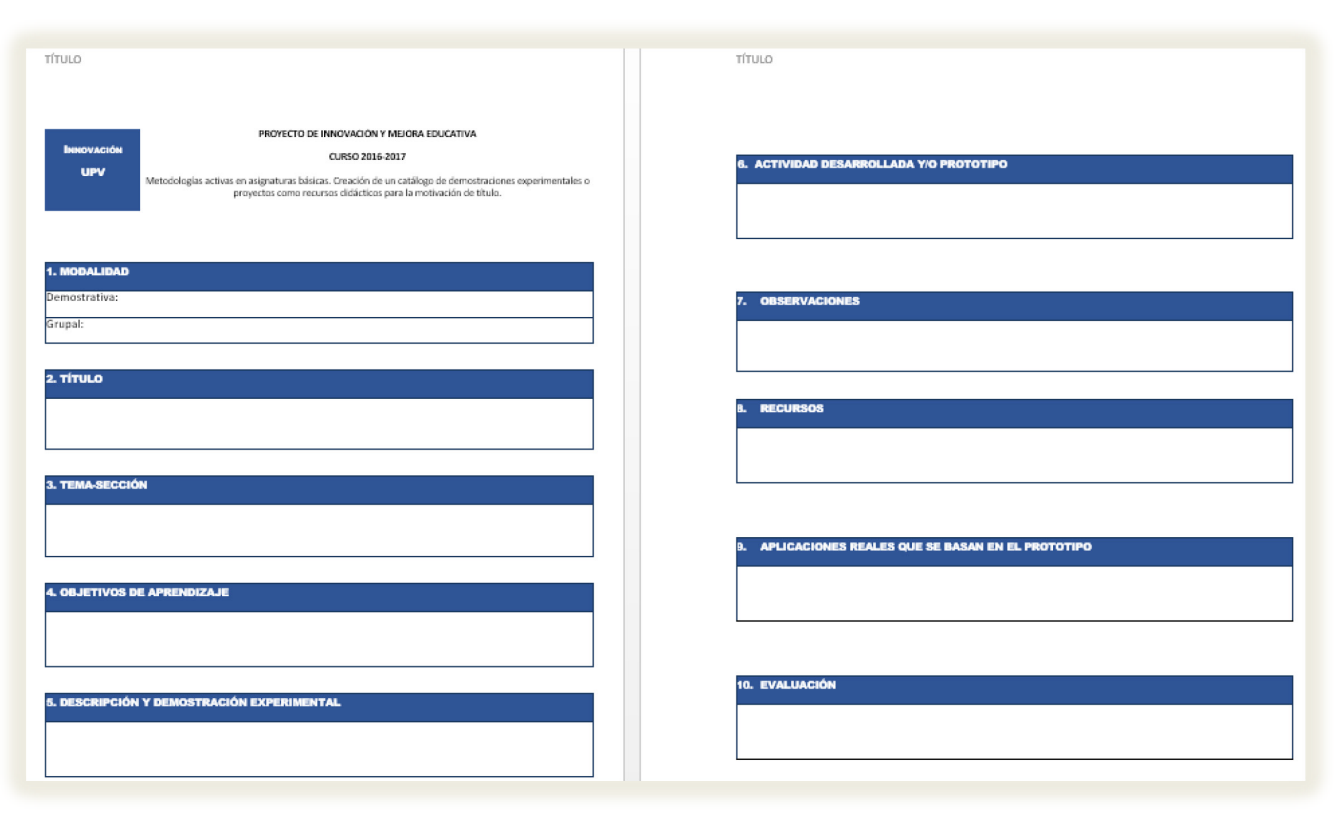

Fig. 2 Ficha a rellenar y sus apartados

El contenido de este fichero se distribuye en 10 apartados:

Apartado 1 "Modalidad"; Cabe destacar que el profesor podrá desarrollar dos tipos de actividades: una práctica demostrativa o una actividad grupal o pequeño proyecto en clase. Esto vendrá indicado en el apartado 1.

Apartado 2 "Título"

Apartado 3 "Tema-Selección”

Apartado 4 "Objetivo de aprendizaje"

Apartado 5 "Descripción y demostración experimental"

Apartado 6 "Actividad desarrollada y/o prototipo"

Apartado 7 "Observaciones"

Apartado 8 "Recursos"; material generado, en el caso de tratarse de una práctica demostrativa, cada profesor deberá crear el material por triplicado. De este modo, en cada campus se dispondrá de un prototipo para poder llevarlo a clase.

Apartado 9 "Aplicaciones reales que se basan en el prototipo"

Apartado 10 "Evaluación”; el profesor planteará un posible modo de evaluación, y en el caso de escoger rúbricas se puede incluir alguna pregunta para que el profesor pueda evaluar alguna de las competencias transversales asignadas como punto de control en sus asignaturas. En el siguiente apartado de "Resultados" se presenta un ejemplo completo de una de estas

(cc)) EY-NG-ND 2017, Universitat Politècnica de València 
fichas que es uno de los pequeños proyectos prototipos usados en clase y desarrollado por los alumnos.

\section{Resultados}

A modo de resultados presentamos algunos ejemplos de los prototipos ya diseñados, así como un ejemplo de una de las fichas asociada a un pequeño proyecto.

\subsection{Prototipos diseñados}

Como hemos mencionado previamente, uno de los objetivos del proyecto es generar un catálogo de demostraciones experimentales y sus correspondientes materiales asociados (prototipo, vídeos y cuestionarios o rúbricas) que permitan a profesores y estudiantes ver y pensar los fenómenos físicos de forma permanente y usarlos para impartir docencia en las asignaturas de Física tanto en clase de teoría como en problemas. Las imágenes de la Tabla 1 muestran algunos de los prototipos ya diseñados.

\section{Tabla 1: Ejemplos de prototipos}

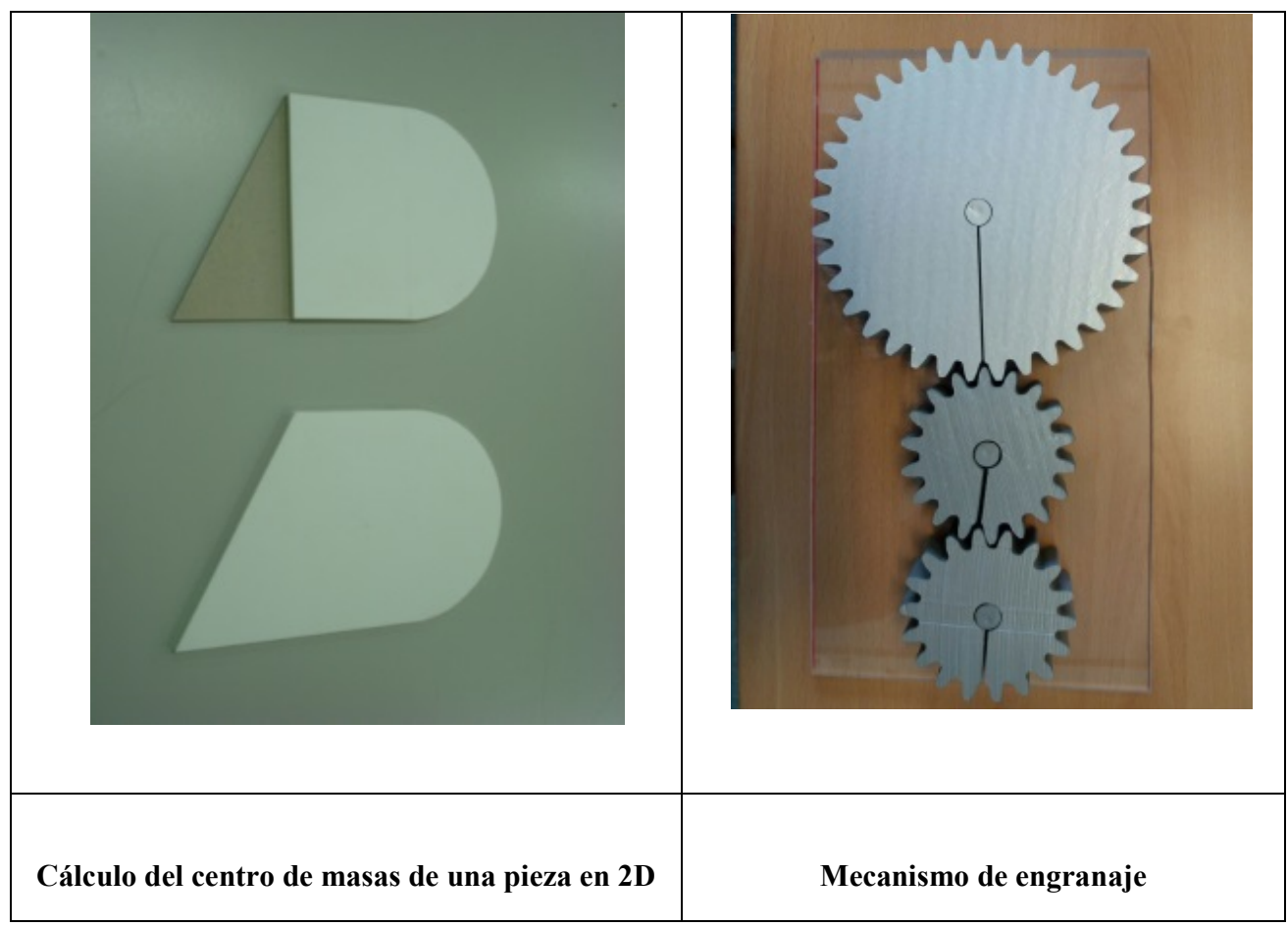




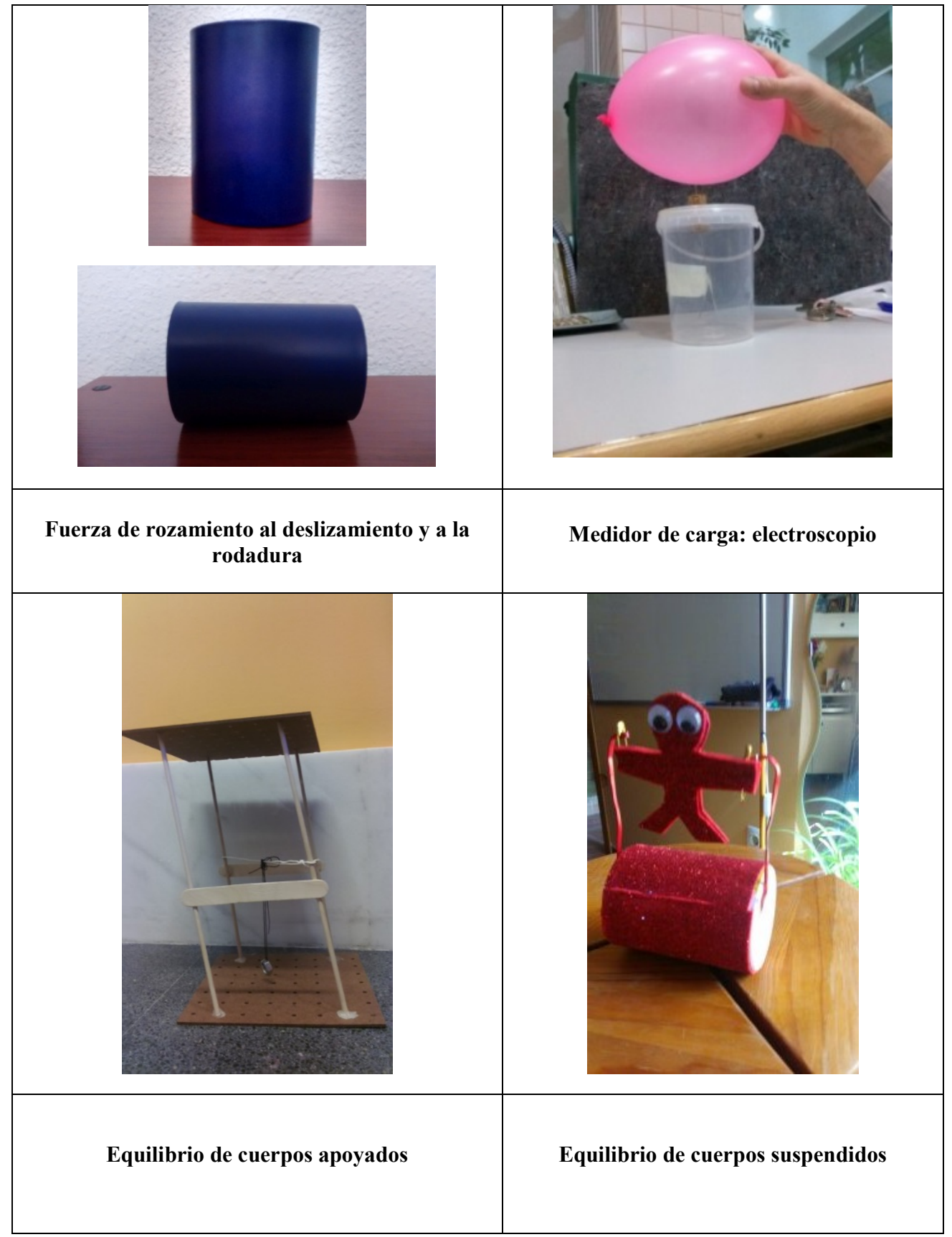

A continuación mostramos un ejemplo de una de las fichas subida al Google Drive. Se trata de una actividad grupal en la que los alumnos crean sus propios prototipos. Esta actividad ha servido como experiencia piloto y nos han permitido comprobar que los alumnos responden con alta motivación y grado de implicación a estas actividades.

(c) ) EY-NG-ND 2017, Universitat Politècnica de València 


PROYECTO DE INNOVACIÓN Y MEJORA EDUCATIVA
CURSO 2016-2017
INNOVACIÓN
UPV \begin{tabular}{c}
$\begin{array}{c}\text { Metodologías activas en asignaturas básicas. Creación de un catálogo de } \\
\text { demostraciones experimentales o proyectos como recursos didácticos } \\
\text { para la motivación de título. }\end{array}$ \\
\hline 1. MODALIDAD \\
\hline Demostrativa: \\
\hline Grupal: $\quad \mathrm{X}$ \\
\hline
\end{tabular}

\section{TÍTULO}

Centro de masas de una pieza en 2D

\section{TEMA-SECCIÓN}

Estática. Centro de gravedad, centro de masas y centroide. Teoremas de Pappus-Guldin.

\section{OBJETIVOS DE APRENDIZAJE}

El alumnado será capaz de:

1. Diferenciar los conceptos de centro de gravedad, centro de masas y centroide.

2. Calcular numéricamente y experimentalmente (método de la plomada) el centro de masas de una pieza en $2 \mathrm{D}$.

3. Aplicar los teoremas de Pappus-Guldin.

4. Ver la relación que hay entre el equilibrio de una pieza y su centro de masas. 


\section{DESCRIPCIÓN Y/O DEMOSTRACIÓN EXPERIMENTAL}

Algunos sistemas continuos en 2D se pueden descomponer en piezas más simples (ver Figura 1) de modo que podemos reducir un sistema continuo a un sistema discreto:

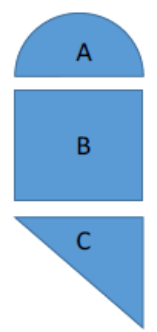

Figura 1: Pieza continua descompuesta en tres subpiezas A, B y C.

El cálculo del Centro de Gravedad (CG) /Centro de Masas ( CM)/Centroide (C) se obtiene a partir de una media ponderada según la gravedad ( CG) , la masa ( CM) o el área (C) de las coordenadas geométricas de las subpiezas:

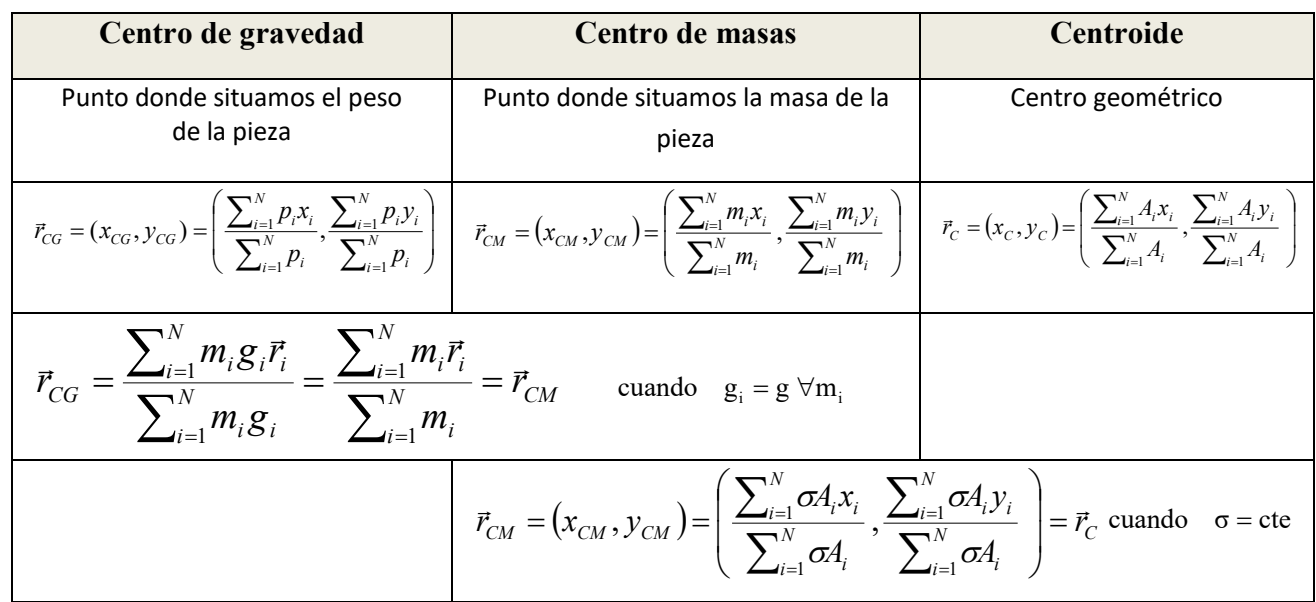

Para encontrar las coordenadas geométricas $(x, y)$ de alguna de las subpiezas puede ser útil usar los teoremas de Papuss-Guldin los cuales están enunciados para el centroide:

\section{$1^{\text {er }}$ TEOREMA DE PAPPUS-GULDIN}

“El área lateral A que engendra una línea de longitud $\mathrm{L}$, al girar alrededor de un eje $\mathrm{X}$ contenido en su plano y que no la corte es igual al producto de la longitud de la línea por la longitud de la circunferencia que describe su centroide en su giro alrededor del eje $\mathrm{X}^{\prime \prime}$

$$
\mathrm{A}=2 \pi \mathrm{y}_{\mathrm{C}} \mathrm{L}
$$

\section{$2^{\text {do }}$ TEOREMA DE PAPPUS-GULDIN}

"El volumen $V$ que engendra una superficie plana de área $A$, al girar alrededor de un eje $X$ contenido en su plano y que no la corte es igual al producto de la superficie que gira por la longitud de la circunferencia que describe su centroide en su giro alrededor del eje $X^{\prime \prime}$

$$
\mathrm{V}=2 \pi \mathrm{y}_{\mathrm{C}} \mathrm{A}
$$




\section{ACTIVIDAD DESARROLLADA}

\section{Material}

1. Distintos materiales: cartulinas, madera, plásticos, etc...

2. Tijeras

3. Pagamento

4. Hilo

5. Regla, escuadradas

\section{Descripción}

1. Pedir a los alumnos que se distribuyan en grupos de 3 o 4 personas.

2. Diseñar una pieza $2 \mathrm{D}$ (formada por cuadrados, rectángulos, círculos, semicírculos, triángulos,...) que pueda reducirse a un sistema discreto y construirla por duplicado con materiales iguales y distintos (ver Figura 2).

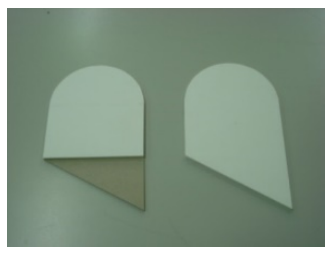

Figura 2 : Pieza formada por un semicirculo, un rectángulo y un triángulo.

3. Calcular numéricamente el centro de masas de la pieza homogénea, indicando claramente la posición del sistema de referencia utilizado: orientación de ejes OX y OY y origen del sistema de referencia.

4. Calcular el centro de masas experimentalmente usando el método de la plomada para la figura homogénea y la heterogénea (ver Figura 3). Obtener las coordenadas de los puntos de intersección de las rectas trazadas a partir de la plomada.
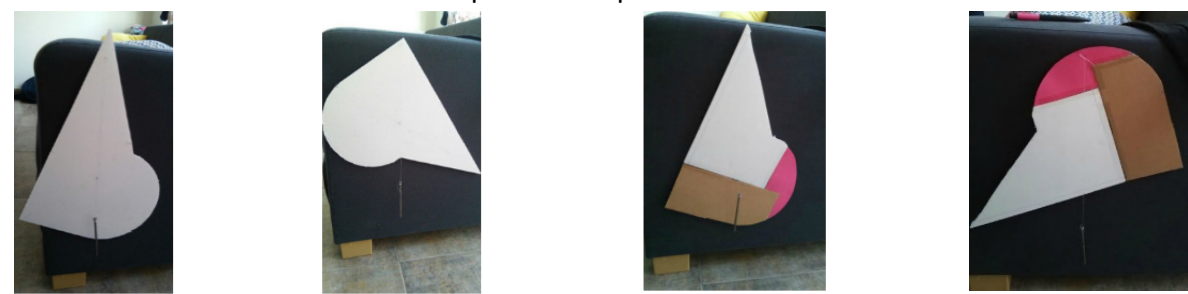

Figura 3: Pieza homogénea y heterogénea colgada de dos puntos diferentes y sus plomadas.

5. Rellenar la Tabla 1:

\begin{tabular}{|c|c|c|}
\hline Centroide calculado & $\begin{array}{c}\text { Centro de gravedad experimental } \\
\text { de la pieza homogénea }\end{array}$ & $\begin{array}{c}\text { Centro de gravedad experimental } \\
\text { de la pieza heterogénea }\end{array}$ \\
\hline & & \\
\hline
\end{tabular}

Tabla 1: Valores de $C$ teórico y del CM experimental de la pieza homogénea y heterogénea.

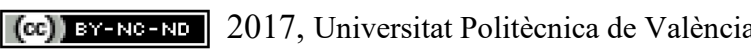


6. Comprobar el equilibrio haciendo girar las piezas homogénea y heterogénea apoyando su CM sobre un dedo (ver Figura 4).

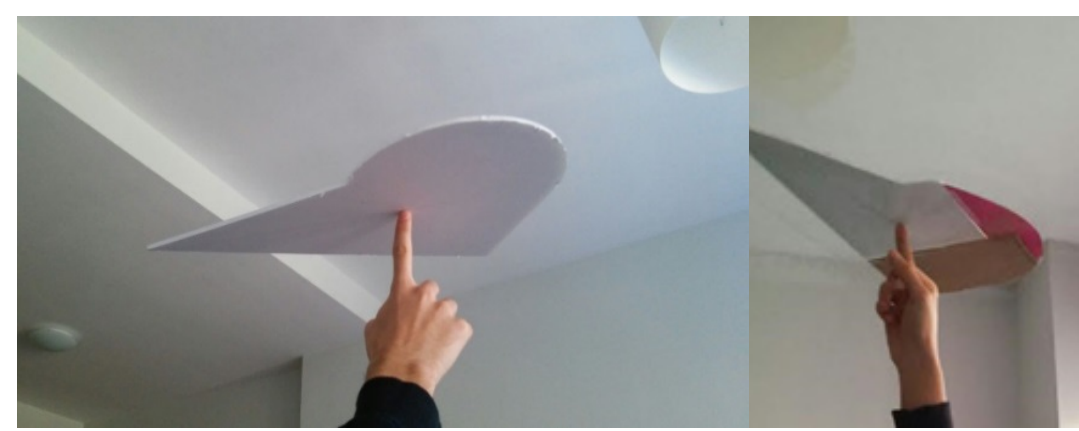

Figura 4: Piezas homogénea y heterogénea con $\mathrm{CM}$ indicado por el dedo.

7. Los alumnos entregan el cálculo realizado y algunos de ellos lo realizan en la pizarra (se pueden escanear todos los ejercicios y subirlos a una plataforma digital para que vean más ejemplos).

8. Se exponen todas las piezas colocándolas juntas (ver Figura 5) en algún lugar de la clase al terminar la sesión:

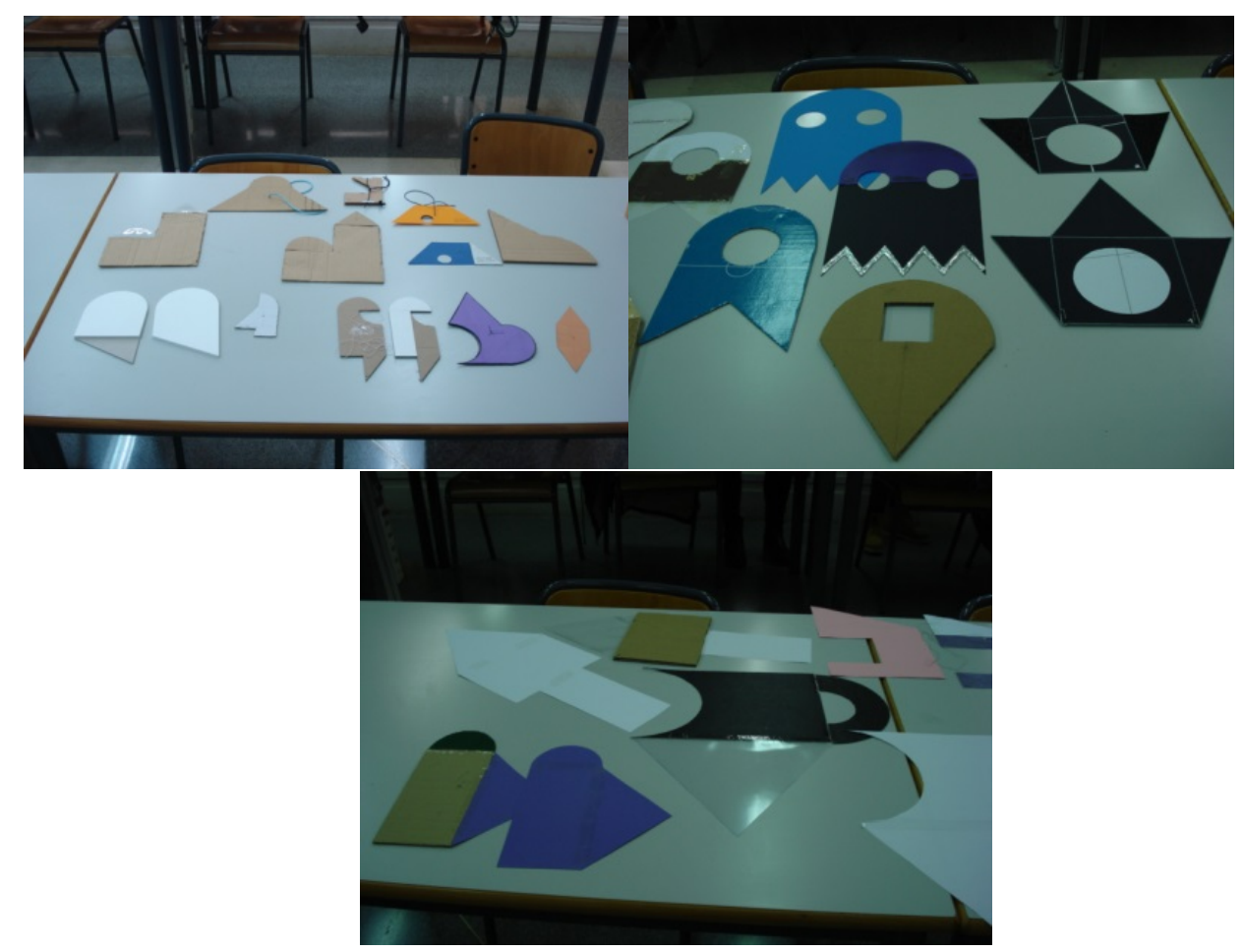

Figura 5: Muestrario de piezas diseñadas por los alumnos.

(c) B EY-NG-ND 2017, Universitat Politècnica de València 


\section{OBSERVACIONES}

1. Si no se usan materiales con densidades de masa muy distintas en el diseño de la pieza no homogénea, el cambio en la posición del CM es muy pequeño y difícil de apreciar usando el método de la plomada.

2. La actividad funciona mejor si se les pide a los alumnos que vengan a clase con las piezas ya diseñadas y con el cálculo del centroide realizado teóricamente.

\section{RECURSOS}

Se generan muchas figuras distintas una por grupo. Y se les puede pedir que cedan alguna.

\section{APLICACIONES REALES QUE SE BASAN EN EL PROTOTIPO}

Ejemplo del centro de gravedad:

El péndulo físico.

Ejemplo del centro de masa:

El estudio de la traslación y la rotación de un sólido rígido homogéneo o heterogéneo respecto a su centro de masas y al eje de rotación que pasa por él.

\section{EVALUACIÓN}

a) Entregar los cálculos del centroide de la pieza diseñada por cada grupo.

b) Entregar la tabla 1 cumplimentada y una valoración de los resultados obtenidos.

c) Entregar por escrito la explicación de la diferencia entre centro de gravedad, centro de masas y centroide.

Fig. 3 Ejemplo de ficha ya completada

\section{Conclusiones}

En este trabajo hemos mostrado cómo desarrollar metodologías activas en asignaturas básicas basadas en la creación de un catálogo de demostraciones experimentales o proyectos como recursos didácticos cuyo objetivo es motivar, por un lado, a los alumnos mejorando el aprendizaje y la comprensión de los modelos teóricos y, por otro lado, a los profesores para mejorar los resultados docentes. La metodología todavía no ha podido ser implementada totalmente en el aula, ya que el proyecto de innovación docente al que este trabajo hace referencia ha sido concedido ya comenzado el curso 2016-2017. Hasta la fecha hemos podido organizar recursos, distribuir carga de profesorado y empezar a disponer de los primeros elementos del catálogo de experimentos y realizar alguna experiencia piloto en el aula.

Lo que esperamos tras la puesta en práctica del proyecto es que tanto en el caso de las actividades grupales o pequeños proyectos, como en el caso de llevar a clase un prototipo, 
los alumnos estén más activos y puedan pasar de "simplemente" ver y escuchar a "realmente" experimentar con el prototipo y hasta crear los suyos propios. En algunos casos ya hemos comprobado que este cambio de enfoque hace que el clima de la clase sea muy dinámico y que los alumnos estén realmente más activos e involucrados con la actividad. Esperamos que se consoliden más los conceptos y no sólo se memoricen. Dicho de otro modo, al cumplir el objetivo del proyecto esperamos que se incremente la capacidad del alumno de resolver problemas en los que se les exige que tengan que pensar y comprender y no sólo recordar para poder resolverlos.

Es de destacar que a nosotros, como profesores, también nos motiva este proyecto ya que se nos dota de más herramientas para trabajar con los alumnos en clase. Y lo más importante, esta metodología de trabajo permite que la clase magistral se enriquezca convirtiéndose en una clase magistral dialogada y preguntada en la que los alumnos ganan protagonismo y están más activos (Cury 2007).

Otra conclusión importante que queremos resaltar es el hecho de que organizarnos como proyecto educativo en el que participamos varios profesores de Física que impartimos diferentes asignaturas en distintos campus universitarios y en el que todos participamos de forma activa permite generar un buen número de recursos educativos con un esfuerzo que no es excesivo. En nuestro caso, nos hemos propuesto generar al menos dos prototipos y una actividad grupal por cada profesor este primer año, y como ya hemos mencionado previamente, crear por triplicado los prototipos. Con ello conseguimos que en cada campus podamos disponer de todos los prototipos. También hemos tomado consciencia de cómo el esfuerzo colaborativo entre profesores - hoy en día facilitado por los recursos tecnológicos de que se disponen - es una herramienta que se puede explotar, de modo que se consiga mejorar los resultados docentes con un esfuerzo razonable. Finalmente resaltar que la situación que a nivel más profundo se pretende corregir es la de fomentar la capacidad de sorpresa ante los fenómenos naturales y articular el proceso de observación racional de modo que el alumno sea capaz de emitir conclusiones en términos de los modelos físicos que se estudian en el ámbito teórico. Dicho de otro modo, entrenarles en el uso y comprensión del método científico.

\section{Referencias}

ALBA J., TORREGROSA C., DEL REY R. (2015) Aprendizaje basado en proyectos: Primera experiencia en la asignatura de Física del Grado en Ingeniería de Telecomunicación, Sonido e Imagen. Universitat Politècnica de València Congreso IN-RED (2015)

APARICIO, F., GONZALEZ, R. M. Y SOBREVILA, M. A. (2005). Formación de Ingenieros. Objetivos, métodos y estrategias. Instituto de Ciencias de la Educación, UPM.

CALVO, I., LOPEZ-GUEDE, J.M. Y ZULUETA, E. (2010). Aplicando la metodología Project Based Learning en la docencia de Ingeniería Técnica en Informática de Gestión, Revista de Formación e Innovación Educativa Universitaria. Vol. 3, No 4, 166-181

CURY, A. (2007). Padres Brillantes, Maestros Fascinantes. Brasil, Planeta. ISBN: 9788408063605

(c) J EY-NG-ND 2017, Universitat Politècnica de València 
DIAZ M. (2007) fq-experimentos. Experimentos caseros de Física y Química.

$<$ http://fq.experimentos.blogspot .com.es/> [Consulta: 29 de mayo de 2017]

FERRER C. (2012) Demostraciones experimentales de Física para el aula. $<$ http://fisicademos.blogs.uv.es/?page_id=29> [Consulta: 29 de mayo de 2017]

GONZÁLEZ, J. y WAGENAAR, R. (2003): Tuning Educational Structures in Europe. Informe Final - Proyecto Piloto, Fase 1, Bilbao, Universidad de Deusto.

VILAPLANA, R. GOMIS O. AND HYDER A. (2011) "Working in terms of competences: activities designed using active methodologies". Safeeullah Soomro New Achievements in Technology, Education and Development. Croatia: In-Tech. Cap. 22, Pags: 352-372. 